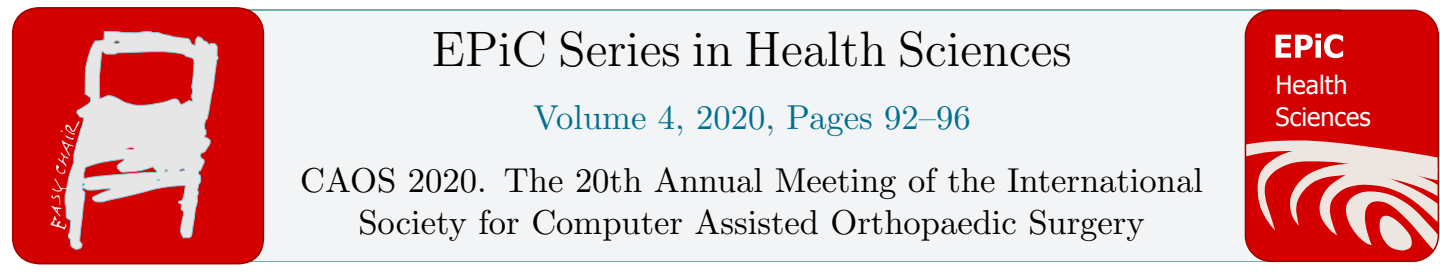

\title{
Alternative Anatomical Landmarks for Anterior Pelvic Plane Determination *
}

\author{
Aziliz Guezou-Philippe ${ }^{12}$, Guillaume Dardenne ${ }^{13}$, Asma Salhi ${ }^{14}$, \\ Valérie Burdin ${ }^{14}$, Christian Lefèvre ${ }^{123}$, and Eric Stindel ${ }^{123}$ \\ 1 Laboratory of Medical Image Processing (LaTIM) - INSERM UMR 1101 - SFR IBSAM, \\ Brest, France \\ 2 University of Western Brittany (UBO), Brest, France \\ 3 Regional University Hospital Center of Brest (CHRU), Brest, France \\ 4 IMT Atlantique, Brest, France \\ aziliz.guezouphilippe@univ-brest.fr
}

\begin{abstract}
The anterior pelvic plane (APP) defined by both anterior superior iliac spines (ASIS) and the pubic symphysis (PS), is used as reference for cup orientation during total hip arthroplasty (THA). However, acquiring the PS and the contralateral ASIS during the intervention with the patient in lateral decubitus position, can be challenging due to the medical devices and the patient abdominal apron. The goal of this study is to find more easily accessible anatomical landmarks, useful for the APP acquisition. Thus we propose to study the variability of the pelvis anatomy in order to identify which landmarks vary with the APP. We built a statistical shape model (SSM) of the pelvis and studied the variability of APP orientation when deforming the SSM along its variation modes. We computed the APP inclination for each deformation and modeled linear relations between the APP inclination and the deformation along the variation modes. We found that the variability in APP inclination is mainly due to 3 variation modes that deform the iliac crest (IC), the posterior superior and anterior inferior iliac spines (PSIS, AIIS). Acquiring those three anatomical landmarks (IC, PSIS and AIIS) with the ipsilateral ASIS, could be a solution to determine more easily the APP for THA in lateral decubitus.
\end{abstract}

\section{Introduction}

Cup orientation is a critical stage in total hip arthroplasty (THA) because of post-operative risks of impingement and dislocation [1]. The commonly accepted safe-zone of Lewinnek [2] recommends to orientate the cup in respect to the anterior pelvic plane (APP), defined by both anterior superior iliac spines (ASIS) and the pubic symphysis (PS). While this safe-zone is nowadays being questioned [3], the APP is still used as reference plane during the surgery.

*This study received funding from the endowment fund INNOVEO of the University Hospital of Brest and the Brittany region.

F. Rodriguez Y Baena and F. Tatti (eds.), CAOS 2020 (EPiC Series in Health Sciences, vol. 4), pp. 92-96 
Navigation solutions exist to help surgeons in identifying the APP. However, for THA in lateral decubitus, the identification of APP landmarks can be very challenging due to the patient abdominal apron covering the PS and the medical devices blocking access to the contralateral ASIS. The goal of this study is to find new anatomical landmarks, more easily accessible than the PS and the contralateral ASIS, to use for APP computation.

To identify such landmarks, we propose to use a statistical shape model (SSM) to analyze the pelvis anatomy variability and to identify which anatomical landmarks vary with the APP inclination. We will first detail the construction of our SSM, then evaluate its quality, and finally study the variability of the APP inclination when the SSM is being deformed along its variation modes.

\section{Materials and Methods}

\section{$2.1 \quad$ Dataset}

We used 40 male CTscans obtained from anonymized datasets available online [4, 5]. Mean age was $54.2 \pm 19.5$ years old and mean BMI was $25.4 \pm 4.3 \mathrm{~kg} / \mathrm{m}^{2}$. Once segmented, the pelvis models were remeshed to 24.000 vertices, to standardize the dataset.

\subsection{Model Construction}

To build the SSM, we generated an unbiased reference mesh with a variant of the IMCP-GMP algorithm [6]. This unbiased reference was then warped to each pelvis in the dataset with the CPD algorithm [7], to establish dense correspondence between meshes. We finally applied a principal component analysis on the dataset in correspondence using Scalismo [8], and obtained a mean mesh and variation modes.

\subsection{Model Evaluation}

CPD fitting quality. The correspondences quality has been assessed computing the mean point-to-mesh distance between the original pelvis meshes and their CPD fits.

Model metrics. As described by Davies et al. in [9], we used the three following metrics to evaluate our model: compactness, generality and specificity.

Compactness illustrates how well the model can describe the dataset shape variability with the fewest variation modes. It corresponds to the percentage of the dataset shape variability represented by the model using $N$ variation modes.

Generality describes how well the model can fit new shapes using $N$ variation modes. We evaluated it through a leave-one-out evaluation and computed the mean error between the fitted model and the targeted mesh.

Specificity describes how well the model can generate new shapes of the same type of the original dataset, using $N$ variation modes. We evaluated it generating randomly 100 meshes from the model and computing the mean point-to-mesh distance between the generated mesh and its closest mesh in the dataset.

\subsection{APP variability when deforming the SSM}

Each mode of variation $M_{i}$ is characterized by its mean $\mu_{i}$ and its standard deviation $\sigma_{i}$. Deformations of the mean shape are generally considered realistic when the variation along the mode $M_{i}$ is in the range of $\mu_{i} \pm 3 \sigma_{i}$. 
For each variation mode $M_{i}$, we deformed the model along $M_{i}$ between $-3 \sigma_{i}$ and $+3 \sigma_{i}$ by steps of $0.1 \sigma_{i}$ and computed the APP inclination at every deformation step (angle between the APP and the vertical in the sagittal plane). We then modeled the APP inclination as a linear function of the deformation along the variation mode $M_{i}$.

\section{$3 \quad$ Results}

\subsection{Model Evaluation}

CPD fitting quality. The average CPD fitting error was $1.34 \pm 0.11 \mathrm{~mm}$.

Model metrics. The obtained values for compactness, generality and specificity are detailed in Table 1.

\begin{tabular}{l||c|c|c|c|c|c|c}
\hline Metric $\quad N$ & 1 & 6 & 11 & 16 & 19 & 26 & 40 \\
\hline \hline Compactness & $43 \%$ & $80 \%$ & $90 \%$ & $95 \%$ & $97 \%$ & $99 \%$ & $100 \%$ \\
\hline Generality (mm) & 3.63 & 2.67 & 2.32 & 2.16 & 1.94 & 1.72 & 1.56 \\
\hline Specificity (mm) & 2.46 & 3.14 & 3.22 & 3.22 & 3.34 & 3.40 & 3.34 \\
\hline
\end{tabular}

Table 1: Model metrics using the first $N$ variation modes

\subsection{APP variability when deforming the SSM}

The first 5 variation modes represented respectively $43 \%, 16 \%, 8 \%, 7 \%$ and $4 \%$ of the datasest variability. Deformations of the SSM along those 5 modes are illustrated in Figure 1.

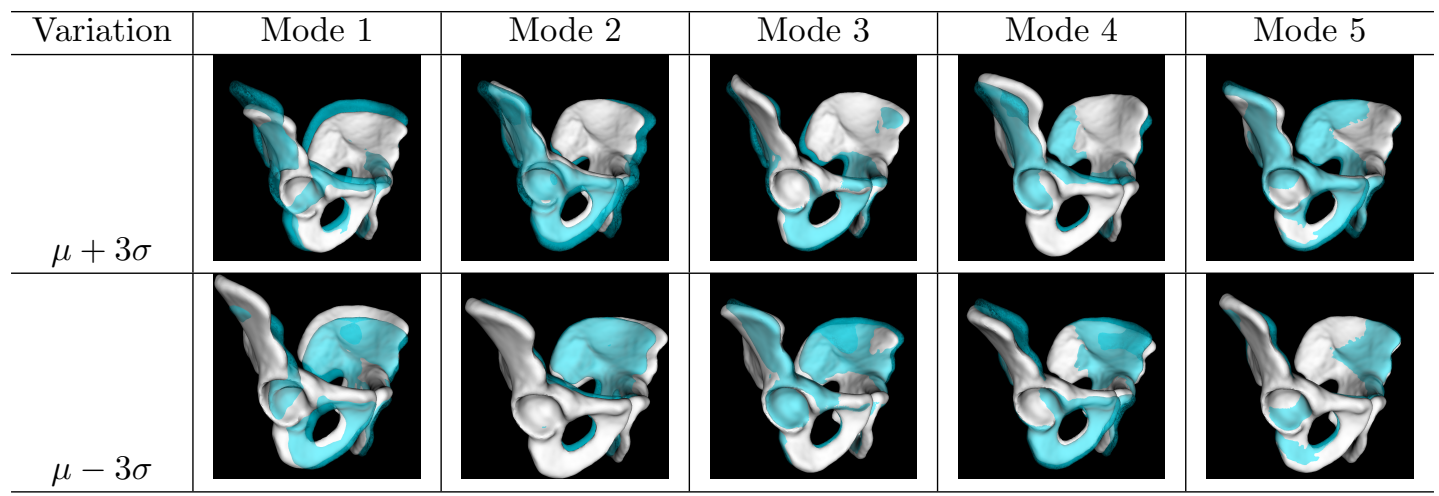

Figure 1: Deformations of the SSM along the first 5 modes of variation; Blue shape is the mean shape $(\mu)$; White shape is the deformed shape between -3 and +3 standard deviation $(\sigma)$

All models of the APP inclination as linear function of the deformation along the variation modes were statistically significant $(\mathrm{p}$-value $<0.01)$, except for the $40^{\text {th }}$ variation mode.

Only five variation modes, the $3^{\text {rd }}, 4^{\text {th }}, 5^{\text {th }}, 12^{\text {th }}$ and the $14^{\text {th }}$, had strong impact on the APP inclination, making it vary by more than $6^{\circ}\left(8^{\circ}, 9^{\circ}, 9^{\circ}, 6^{\circ}\right.$ and $8^{\circ}$ for each variation mode respectively). Those modes represented respectively $8 \%, 7 \%, 4 \%, 1 \%$ and $1 \%$ of the dataset variability and deformed the pelvis SSM at the ASIS, the ischial tuberosities, the iliac crest (IC), the posterior superior iliac spine (PSIS) and the anterior inferior iliac spines (AIIS). 
Four variation modes had medium impact on the APP inclination, making it vary by $4^{\circ}$ approximately. Each of those variation modes represented less than $2 \%$ of the dataset variability.

The remaining variation modes had low impact on the APP variation, making it vary by less than $3^{\circ}$.

\section{Discussion}

We built a SSM of the male pelvis, that has realistic mean shape and deformations, with metrics similar to what can be found in the literature $[10,11]$.

The SSM first two variation modes represented the highest percentage of the dataset variability $\left(43 \%\right.$ and $16 \%$ ) but had a low impact on the APP inclination (less than $3^{\circ}$ ) as they deformed the pelvis shape in size and in width. The $3^{r d}, 4^{\text {th }}$ and $5^{\text {th }}$ variation modes represented lower percentages of the dataset variability but had the highest impact on the APP (more than $8^{\circ}$ ), deforming the pelvis SSM at the ASIS, the ischial tuberosities, the IC, the PSIS and the AIIS. Those last 3 landmarks seem interesting for APP determination as they can be acquired by ultrasound (US) imaging.

Some authors already used the IC and PSIS as landmarks for the APP determination [12, 13], acquiring them using US imaging and tracked pointers. However they did not consider the nonaccessibility of the contralateral landmarks due to the patient position on the operative table.

In the future, we will work on acquiring the ASIS, PSIS, AIIS and IC from the operated side of the pelvis, using a tracked US probe [14, 15], to easily determine the APP inclination for THA in lateral decubitus position.

\section{References}

[1] R. Biedermann, A. Tonin, M. Krismer, F. Rachbauer, G. Eibl, and B. Stöckl. Reducing the risk of dislocation after total hip arthroplasty. The Journal of Bone and Joint Surgery. British volume, 87-B(6):762-769, 2005.

[2] G E Lewinnek, J L Lewis, R Tarr, C L Compere, and J R Zimmerman. Dislocations after total hip-replacement arthroplasties. The Journal of Bone \& Joint Surgery, 60(2):217-220, 1978.

[3] Lawrence D Dorr and John J Callaghan. Death of the Lewinnek "Safe Zone". The Journal of Arthroplasty, 34(1):1-2, 2019.

[4] Michael Kistler, Serena Bonaretti, Marcel Pfahrer, Roman Niklaus, and Philippe Büchler. The virtual skeleton database: An open access repository for biomedical research and collaboration. Journal of Medical Internet Research, 15(11):e245, 2013.

[5] University of iowa magnetic resonance research facility. https://mri.radiology.uiowa.edu/ images_main.html, last viewed January 2020.

[6] Tinashe Mutsvangwa, Valerie Burdin, Cedric Schwartz, and Christian Roux. An Automated Statistical Shape Model Developmental Pipeline: Application to the Human Scapula and Humerus. IEEE Transactions on Biomedical Engineering, 62(4):1098-1107, 2015.

[7] Andriy Myronenko and Xubo Song. Point Set Registration : Coherent Point Drift. IEEE Transactions on Pattern Analysis and Machine Intelligence, 32(12):2262-2275, 2010.

[8] Scalismo - scalable image analysis and shape modeling. Available online at: http://github.com/ unibas-gravis/scalismo, 2016.

[9] Rhodri Davies, Carole Twining, and Chris Taylor. Statistical Models of Shape: Optimisation and Evaluation. Springer Science \& Business Media, 2008.

[10] Sebastian Meller and Willi A. Kalender. Building a statistical shape model of the pelvis. International Congress Series, 1268(C):561-566, 2004. 
[11] Peter Vanden Berghe, Jan Demol, Frederik Gelaude, and Jos Vander Sloten. Virtual anatomical reconstruction of large acetabular bone defects using a statistical shape model. Computer Methods in Biomechanics and Biomedical Engineering, 20(6):577-586, 2017.

[12] Dean C. Barratt, Carolyn S.K. Chan, Philip J. Edwards, Graeme P. Penney, Mike Slomczykowski, Timothy J. Carter, and David J. Hawkes. Instantiation and registration of statistical shape models of the femur and pelvis using 3D ultrasound imaging. Medical Image Analysis, 12(3):358-374, 2008.

[13] Steffen Schumann, Lutz-P. Nolte, and Guoyan Zheng. Determination of pelvic orientation from sparse ultrasound data for THA operated in the lateral position. The International Journal of Medical Robotics and Computer Assisted Surgery, 8(1):107-113, 2012.

[14] Guillaume Dardenne, Jean-Philippe Pluchon, Hoel Letissier, Aziliz Guezou-Philippe, Romain Gerard, Christian Lefèvre, and Eric Stindel. Accuracy and Precision of an Ultrasound-Based Device to Measure the Pelvic Tilt in Several Positions. Journal of Ultrasound in Medicine, oct 2019.

[15] Mateo Villa, Guillaume Dardenne, Maged Nasan, Hoel Letissier, Chafiaa Hamitouche, and Eric Stindel. Fcn-based approach for the automatic segmentation of bone surfaces in ultrasound images. International Journal of Computer Assisted Radiology and Surgery, 13(11):1707-1716, 2018. 